Relations industrielles

Industrial Relations

\title{
Indexation des salaires et paix industrielle
}

Jean-Michel Cousineau

Volume 34, numéro 4, 1979

URI : https://id.erudit.org/iderudit/029015ar

DOI : https://doi.org/10.7202/029015ar

Aller au sommaire du numéro

Éditeur(s)

Département des relations industrielles de l'Université Laval

ISSN

0034-379X (imprimé)

1703-8138 (numérique)

Découvrir la revue

Citer cet article

Cousineau, J.-M. (1979). Indexation des salaires et paix industrielle. Relations industrielles / Industrial Relations, 34(4), 793-798.

https://doi.org/10.7202/029015ar

Tous droits réservés (c) Département des relations industrielles de l'Universite Laval, 1979
Ce document est protégé par la loi sur le droit d'auteur. L'utilisation des services d'Érudit (y compris la reproduction) est assujettie à sa politique d'utilisation que vous pouvez consulter en ligne.

https://apropos.erudit.org/fr/usagers/politique-dutilisation/ 


\title{
Indexation des salaires et paix industrielle
}

\author{
Jean-Michel Cousineau
}

Cette note a pour but de présenter les résultats d'une analyse empirique de l'effet de l'indexation des salaires sur la fréquence des arrêts de travail au Canada, dans les grandes conventions collectives du secteur privé. L'indexation des salaires peut influencer les arrêts de travail de deux façons. D'une première façon, elle peut en diminuer le nombre si elle permet d'éviter des pertes de pouvoir d'achat aux travailleurs. Nous chercherons à savoir si le fait d'avoir obtenu des augmentations de salaire inférieures à l'augmentation du coût de la vie dans une précédente convention, donne lieu à une probabilité de grève plus élevée lors du renouvellement de cette convention. Dans la mesure où une clause d'indexation est efficace et permet d'éliminer ce problème, elle réduira le nombre de grèves. Par ailleurs, on peut se demander si l'acquisition, par un syndicat, d'une telle clause ne constitue pas, en soi, un facteur de grève. Dans cette éventualité, ce second effet viendrait annuler le premier. Il s'agit donc de connaître l'effet net des clauses d'indexation sur les grèves, puisqu'un effet tend à en diminuer le nombre et un autre pourrait l'accroître. Nous étudierons successivement l'un et l'autre effet.

\section{L'INCIDENCE DES PERTES DE SALAIRE REELL SUR LA FRÉQUENCE DES GRÈVES}

Dans la mesure où l'inflation est incorrectement anticipée par les travailleurs ou leurs représentants syndicaux, comme cela a été fréquemment le cas plus particulièrement entre 1973 et 1975 au Canada (voir tableau 1), une convention collective peut donner lieu à des augmentations de salaire inférieures à l'évolution du coût de la vie telle que mesurée par les variations en pourcentage de l'indice général des prix à la consommation. Pareille situation est susceptible de hausser les demandes syndicales tout en laissant inchangées les offres patronales.

- COUSINEAU, Jean-Michel, professeur, École de relations industrielles, Université de Montréal.

** L'auteur remercie Travail Canada de la subvention qui lui a été accordée pour effectuer cette recherche. Il remercie également Denis Grégoire pour son aide technique ainsi que François Dussault, économiste-chercheur à l'Université de Montréal et le profeseur François Vaillancourt du Département de Sciences économiques de cette même université pour leurs suggestions. 
Tableau 1

Renouvellement de conventions et pertes de salaire réel, grandes conventions collectives, Canada, 1973-1975

\begin{tabular}{cccc} 
Année & $\begin{array}{c}\text { Proportion des } \\
\text { conventions avec } \\
\text { pertes (\%) }\end{array}$ & $\begin{array}{c}\text { Proportion des } \\
\text { employés touchés }\end{array}$ & $\begin{array}{c}\text { Pertes de salaire } \\
\text { réel en pourcentage }\end{array}$ \\
1973 & 19.1 & 18.6 & -1.5 \\
1974 & 43.6 & 30.9 & -3.7 \\
1975 & 34.2 & 33.1 & -4.8 \\
\hline
\end{tabular}

Source: Cousineau et Lacroix (1977).

Du point de vue syndical, il paraît des plus légitimes de fonder ses réclamations sur un tel manque à gagner. Du point de vue patronal, toutefois, la baisse dans les coûts du travail peut avoir servi à un accroissement de l'embauche si on était en situation d'offre excédentaire sur le marché du travail. Par ailleurs, l'accroissement des profits, si accroissement il y zut - les profits de même que les salaires peuvent avoir diminué en termes réels -, peut avoir été affecté à des projets d'investissements ou à l'achat de matières premières. Enfin, il apparaît plus probable que les calculs de l'entreprise en négociation s'effectuent en fonction des prévisions de ventes et de prix dans le futur plutôt que sur le résultat passé d'une précédente convention collective. Les offres salariales de la partie patronale pourront donc demeurer relativement insensibles au problème de rattrapage. Toutes choses égales par ailleurs, pareille situation est susceptible d'accroître la probabilité de grève.

Pour tester cette hypothèse, il nous faut identifier une variable dépendante ainsi qu'un ensemble de variables indépendantes.

\section{La variable dépendante}

Afin de refléter le mieux possible la probabilité de grève à un niveau agrégé, nous avons choisi de retenir le nombre de grèves dans les grandes conventions collectives en pourcentage du nombre total de négociations salariales par trimestre (TG) ${ }^{1}$. Ces données excluent toutes considérations de grèves en dehors du cadre des négociations. Elles nous permettent aussi de tenir compte du potentiel de grève en tenant compte du nombre de négociations, ce que peu d'études ont pu faire à notre connaissance. Elles permettent, enfin, de faire la distinction importante entre les grèves du secteur privé et celles du secteur public. Parce que les mécanismes de détermination de l'activité de grève ainsi que les contraintes économiques

1 Les grandes conventions sont celles de 500 membres et plus et excluent le secteur de la construction. Données inédites de Travail Canada sur chacune des grandes conventions collectives signées au Canada entre 1967 et 1975 . TG est donc le nombre de cas où il y eut grèves (multiplié par 100) sur le nombre de conventions signées au cours du trimestre. 
diffèrent grandement d'un secteur à l'autre, nous avons exclu de notre analyse les grèves du secteur public (administrations fédérales, provinciales et municipales ainsi que les secteurs de l'éducation et de la santé).

\section{Les variables indépendantes}

À l'instar des travaux de Ashenfelder et Johnson (1969), Cousineau et Lacroix (1976), Skeels (1971), Vanderkamp (1970) et Walsh (1975), nous avons cherché à retenir des indicateurs de l'activité économique et de l'inflation comme variables explicatives de la fréquence des arrêts de travail. Nous avons construit un indice du taux de postes vacants (ITV) retardé d'un trimestre ${ }^{2}$ pour refléter l'évolution de l'activité économique. La variable choisie pour refléter l'inflation a été celle des variations annuelles en pourcentage de l'indice général des prix à la consommation $(\dot{\mathrm{P}}$, données trimestrielles; source: Prix à la consommation et indice des prix, Statistique Canada, cat. no 62-010, trimestriel). À ce modèle standard, nous ajoutons la variable rattrapage ou pertes de salaire réel.

La variable rattrapage $(R)$ est représentée par le pourcentage des conventions collectives renégociées qui faisaient suite à une précédente convention où les gains salariaux s'étaient avérés inférieurs à l'augmentation des prix à la consommation (R). Une convention collective est en situation de rattrapage si son augmentation de salaire s'est avérée inférieure à l'augmentation de l'indice des prix à la consommation (IPC). Nous avons donc calculé la différence entre les augmentations de salaire de chacune des conventions et l'augmentation en pourcentage de l'IPC au cours de la durée du contrat pour connaître le nombre de conventions en situation de rattrapage.

La spécification empirique du modèle peut donc s'écrire

(1) $\mathrm{TG}=\mathrm{a}_{0}+\mathrm{a}_{1}$ ITV $_{-1}+\mathrm{a}_{2} \dot{\mathrm{P}}+\mathrm{a}_{3} \mathrm{R}+\mathrm{u}$

Parce que l'activité économique et l'inflation ont tendance à influencer les demandes et les offres salariales dans le même sens, le signe des coefficients

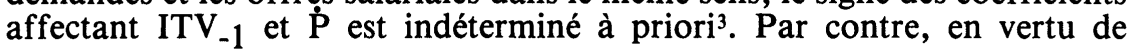
l'analyse précédente, on s'attend à une relation positive entre la variable de grève et la variable de rattrapage.

Les résultats d'estimation, pour la période 1970II-1975IV4, sont les suivants. Les tests «t» apparaissent entre parenthèses sous les coefficients.

2 ITV = I.O.E. X $\frac{\text { PA }}{\mathrm{PA}_{\mathrm{t}}} 1969$ où I.O.E. = indice de l'offre d'emploi (source: Revue économique, Ministère fédéral des Finances, Ottawa, annuel) et $\mathrm{PA}=$ population active (source: La population active, Statistique Canada, cat. no. 71-001). Mentionnons également que l'ITV n'est pas basé sur les données de Statistique Canada sur les postes vacants. Pour la méthodologie associée à l'I.O.E., voir Revue économique, ministère fédéral des Finances, avril 1973, p. 73.

3 Pour une discussion plus élaborée de cette question, voir COUSINEAU et LACROIX (1976).

4 Plusieurs observations ont été perdues au début de la période (1967-1969) parce qu'on ne pouvait se référer à une convention précédente pour calculer l'importance du rattrapage. 


$$
\text { (2) } \begin{aligned}
\mathrm{TG} & =\underset{(7.4)}{43.2-0.476 \mathrm{ITV}_{-1}}+\underset{(5.07)}{3.39 \dot{\mathrm{P}}}+\underset{(4.37)}{0.287} \mathrm{R} \\
\mathrm{R}^{2} & =.684 \overline{\mathrm{R}}^{2}=.631 \mathrm{D} .-\mathrm{W} .=1.38 \\
\text { (3) } \mathrm{TG}= & 35.2-0.365 \mathrm{ITV}_{-1}+\underset{(3.00)}{3.04 \dot{\mathrm{P}}}+\underset{(2.19)}{0.142 \mathrm{R}} \\
\mathrm{R}^{2}= & .744 \overline{\mathrm{R}}^{2}=.634 \text { D.-W. }=1.749
\end{aligned}
$$

L'équation (3) résulte d'une application de la méthode Hildreth-Lu à l'équation (2), pour tenir compte des problèmes d'autocorrélation. Cette correction conduit effectivement au rejet de l'hypothèse d'auto-corrélation des résidus au seuil de $5 \%$. Tel qu'attendu, on y observe une diminution dans la valeur des statistiques «t», mais il reste que chacun des coefficients demeure significatif au seuil de $5 \%$. Les coefficients de la variable inflation et de la variable rattrapage s'avèrent donc significatifs et de signe positif. Par ailleurs, le coefficient de la variable posies vacants est de signe négatif.

En termes d'impact, on trouve à partir de l'équation (3), que chaque variation de 10 points dans l'indice du taux de postes vacants fait varier de façon inverse le taux de grève de 3.65 points en pourcentage. Toutes choses égales par ailleurs, plus la conjoncture s'améliore plus la fréquence des grèves est faible et, inversement, plus la conjoncture se détériore plus la fréquence des grèves s'accroît. Par ailleurs, chaque variation de 1 point de pourcentage dans l'inflation amène une variation correspondante de 3.39 points de pourcentage dans le taux de grève. Plus l'inflation s'accélère, plus la fréquence des grèves s'accroît et, inversement, si l'inflation décélère, la fréquence des grèves a tendance à diminuer. Enfin, chaque variation de 10 points de pourcentage dans le taux de rattrapage amène une variation correspondante de 1.42 points de pourcentage dans le taux de grève. Plus les problèmes de rattrapage sont nombreux plus la fréquence des grèves s'élève et, inversement, moins ils sont nombreux, plus la fréquence des grèves diminue.

Afin de connaître l'importance relative de chaque variable en termes d'impact sur la variable dépendante, nous avons procédé au calcul des coefficients Bêta. Ceux-ci sont de 2.05 pour la variable ITV $_{-1}, 1.86$ pour la variable $\dot{P}$ et .53 pour la variable $R$. L'influence des postes vacants sur le taux de grève semble donc la plus importante. Par contre, si on retient une classification en termes de coefficients de corrélation partielle, entre la variable dépendante et chacune des variables indépendantes, on trouve que $\mathbf{R}$ est la variable la plus importante. Il semble donc que la corrélation entre le taux de grève et le taux de rattrapage est la plus forte, mais que ce sont les postes vacants qui donnent l'impact le plus important. La raison en est que l'impact du rattrapage sur le taux de grève est local; il se concentre principalement sur une sous-période d'observation, la période 73-75. Les postes vacants, pour leur part, ont fluctué sur l'ensemble de la période d'observation. Par contre, les effets locaux du rattrapage s'avèrent très substantiels. En effet, le taux de rattrapage passe de $0 \%$ au début des années 70 , à $50 \%$ en certains trimestres des années 73-75. Cette variation dans le taux de rat- 
trapage peut donc expliquer, à elle seule, une variation de 7 points de pourcentage dans le taux de grève au cours de notre période d'observation. À certains moments, l'incidence du rattrapage sur la fréquence des arrêts de travail peut donc s'avérer très importante.

\section{ACQUISITIONS DE CLAUSES D'INDEXATION ET FRÉQUENCE DES GRÈVES}

Par ailleurs, comme nous l'avons mentionné en introduction, la lutte pour obtenir une clause d'indexation peut elle-même conduire à des conflits industriels plus nombreux. Le problème de l'incidence de la clause d'indexation sur les conflits industriels se pose donc en termes d'impact net. Afin de tester l'incidence de l'acquisition d'une clause d'indexation sur la fréquence des grèves, nous avons ajouté la variable TCI à l'équation (1), cette variable représentant le nombre de conventions indexées en pourcentage du nombre de négociations. Les résultats obtenus, en appliquant la méthode HildrethLu, sont les suivants:

$$
\text { (4) } \begin{aligned}
\mathrm{TG}= & 38.0-\underset{\left(-409 \mathrm{ITV}_{-1}\right.}{ }+\underset{(3.27)}{3.29 \mathrm{P}}+\underset{(2.61)}{0.171} \mathrm{R}-0.084 \mathrm{TCI} \\
\mathrm{R}^{2}= & .766 \mathrm{R}^{2}=.577 \mathrm{D} . \mathrm{W} .=1.81
\end{aligned}
$$

Ce résultat, tout en n'affectant pas significativement les paramètres de ITV $_{-1}$, $\dot{\mathrm{P}}$ et $\mathrm{R}$, nous indique que TCI n'a pas d'influence marquée sur le taux de grève. Il semble donc que l'adoption d'une clause d'indexation ne conduit pas en soi à des arrêts de travail plus nombreux.

\section{CONCLUSION}

En conclusion, il ressort de notre analyse que l'incidence du rattrapage sur la fréquence des arrêts de travail dans les grandes conventions collectives n'a pas été négligeable. Les résultats d'estimation contribuent, dans une certaine mesure, à expliquer l'explosion des arrêts de travail connue au Canada dans les années 74-75. Cette observation semble des plus pertinentes pour les années 79 et 80 vu l'importance qu'a pu prendre le problème du rattrapage en 1978. En effet, la loi des contrôles sur les prix et les revenus contraignant les augmentations de salaire à des pourcentages voisins de $6 \%$ pour cette année, est susceptible d'avoir conduit à de nombreuses situations où les gains salariaux seront inférieurs à la progression du coût de la vie. En date de novembre 1978, la variation annuelle en pourcentage de l'IPC était de $8.8 \%$. Toutes choses étant égales par ailleurs, on peut prévoir une nouvelle poussée dans le taux de grève au Canada, lorsque ces conventions seront renégociées.

L'indexation des salaires ayant une incidence sur de multiples variables on ne peut en apprécier toute la valeur sur la base d'une analyse aussi partielle. Néanmoins, il reste qu'elle peut constituer sinon un facteur de paix industrielle, au moins une protection contre de brusques augmentations dans le nombre d'arrêts de travail. 


\section{BIBLIOGRAPHIE}

ASHENFELTER, O. et G.E. JOHNSON, "Bargaining Theory, Trade Unions, and Industrial Strike Activity", American Economic Review, mars 1969, pp. 35 à 49.

COUSINEAU, Jean-Michel et Robert LACROIX, «Activité économique, inflation et activité de grève», Relations Industrielles/Industrial Relations, Vol. 31, no 3, juillet 1976, pp. 341 à 357 .

COUSINEAU, Jean-Michel et Robert LACROIX, La détermination des salaires dans le monde des grandes conventions collectives: une analyse des secteurs privé et public, Conseil économique du Canada, Approvisionnements et Services Canada, Ottawa, 1977.

HICKS, John R., The Theory of Wages, London, Macmillan, 1963, pp. 136 à 158.

SKEELS, J.W., "Measures of U.S. Strike Activity”, Industrial and Labor Relations Review, Vol. 24, no. 4, juillet 1971 , pp. 515 à 525 .

VANDERKAMP, John, "Economic Activity and Strikes in Canada", Industrial Relations, Vol. 9, no. 2, février 1970, pp. 212 à 230.

WALSH, W.D., "Economic Conditions and Strike Activity in Canada", Industrial Relations, Vol. 14, no. 1, février 1975 , pp. 45 à 55 .

\section{ECOLE DE RELATIONS INDUSTRIELLES DE L'UNIVERSITÉ DE MONTREAL}

\section{Colloques}

- Formes nouvelles de syndicalisme, (5e colloque), 1974, 66 pages.

- L'État et la transformation des relations industrielles au Québec, (6e colloque), 1976, 64 pages.

- Les conflits en milieux de travail, (7e colloque), 1977, 100 pages.

- Pour une meilleure qualité de vie ... les avantages sociaux, (8e colloque), 1978, 96 pages.

- La réforme des lois du travail, (9e colloque), 1979, 124 pages.

\section{Monographles}

- C. D'AOUST et L. LECLERC, La jurisprudence arbitrale québécoise en matière de congédiement, 1978, 181 pages.

- L.P. DAUBIGNEY, Salaire et marché du travail interne d l'entreprise, 1978, 51 pages.

- G. GUERIN, Le système de planification des ressources humaines dans l'entreprise, 1978,95 pages.

Service des publications, Ecole de relations industrielles,

Université de Montréal, Case Postale 6126, Montréal, H3C 3J7, (514)343-7312 